\title{
THE MODE OF ACTION OF ACETYLCHOLINE, ARECOLINE, AND PILOCARPINE ON THE REGENERATION OF VISUAL PURPLE
}

\author{
M. T. PENG AND S. Y. PENG* \\ Department of Physiology, Medical College, National Taiwan University, \\ Taipei, Formosa, China
}

The acceleratory action of pilocarpine on the regeneration of visual purple was described by Kühne and Ayres (1) and was confirmed by Dresser (2), Inatomi (3), and Amenomiya (4). Hosoya (5) extended Kühne's work and reported that not only pilocarpine but also acetylcholine, arecoline and scopolamine have augmentative action on the regeneration of visual purple. But the mode of action of these drugs on the regeneration of visual purple was quite obscure.

Since the excellent work of Morton and his co-workers (6) which defined retinene as vitamin A aldehyde, Morton et al. (7), Wald and Hubbard (8) and Bliss (9) explored the mechanism of the bleaching of retinene to vitamin A. At the same time they studied the regeneration of visual purple with retinal homogenate $(10,11)$ or visual purple solution (12).

Due to these researches we can now presume the mode of action of these drugs on the regeneration of visual purple. There are two possibilities concerning the mode of action of these drugs. The first one is that these drugs augment the regeneration of visual purple directly; in other words, these drugs accelerate the action of retinene reductase or the action of pigment layer factor. The second one is that the action of these drugs is dependent on living process; in other words, the administration of these drugs induces some functional changes in the pigment layer which augment the regeneration of visual purple. If the latter is the case, there are two further possibilities. The first one is that the application of these drugs causes some physical changes, such as the increase of diffusion or permeability, in the pigment layer which facilitate delivery of the substances neccessary for regeneration from pigment layer to retina, and the second one is that some chemical changes, such as increase of secretion or so on, occur in the pigment layer after the injection of these drugs and such changes augment the regeneration.

With these assumptions the mode of action of acetylcholine, arecoline, and pilocarpine was studied.

\section{MATERIALS AND METHODS}

Retinas and pigment layers of dark adapted toads (Bufo melanostictus Schneider) or frogs (Rana tigerina Daudin) were dissected under dim red light

Received for publication March 31, 1954.

*彭明聰, 彭淑媱 
separately, illuminated for 10 minutes with $100 \mathrm{~V}, 250 \mathrm{~W}$ lamp at a distance of $30 \mathrm{~cm}$. through a glass tray of water $(4 \mathrm{~cm}$.) below the lamp for absorbing heat. They were left for 1 to 1.5 hours in the ordinary light at room temperature, to allow retinas or rods adhered to pigment layers to bleache to colorless. Then the retinas and pigment layers were homogenized separately with glass pestles. Homogenate of retinas, homogenate of pigment layers, DPN or DPN supplemented with niacin amide, and Clark-Lubs' buffer $(p H$ 7.0-7.2) were mixed. The final volume of the mixture was $1.0 \mathrm{ml}$. As the coenzyme DPN in a final concentration of $2 \mathrm{mg} / \mathrm{ml}$. was used in the first part of experiment, but afterward DPN in concentration of $0.1 \mathrm{mg} / \mathrm{ml}$. supplemented with $0.03 \mathrm{M}$ of niacin amide was used. After incubation (overnight incubation was used at first, but afterward 6 hours incubation was adopted) in the dark at room temperature, the mixtures were centrifuged and the solid residues were extracted with 0.6 $\mathrm{ml}$. of $1 \%$ digitonin or $2 \mathrm{ml}$. of $2 \%$ sodium glycocholate. The extracts were added with buffer ( $p$ H 9.0) and were examined for the presence of visual purple by measuring the absorption spectra with Beckman spectrophotometer, bleaching in $100 \mathrm{~V}, 100 \mathrm{~W}$ lamp at a distance of $10 \mathrm{~cm}$., remeasuring the absorption spectra. The difference in absorption spectra before and after bleaching gives the amount of regenerated visual purple.

As drugs, acetylcholine chloride (ovisot, Daiichi), arecoline hydochloride (Merck), pilocarpine nitrate (S. B. Penick), DPN (sigma, 90\% purity), and digitonin (Eimer and Amend) were used.

\section{RESULTS}

Regeneration of visual purple with retinal homogenate. Homogenate of 10 whole bleached retinas was added with homogenate of 10 pigment layers supplemented with DPN, and incubated in the dark at room temperature overnight.

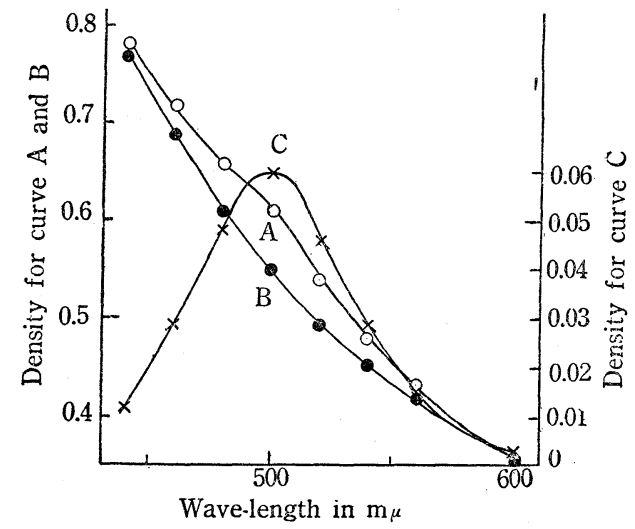

Its differential spectrum before and after bleaching at the range from 440 to $600 \mathrm{~m} \mu$ is shown in fig. 1 . The maximum located at about $500 \mathrm{~m} \mu$ and its form was similar to that of visual purple, so it is safe to say that this is the regenerated visual purple.

FIG. 1. The absorption spectrum of regenerated visual purple.

Curve $A$ is the spectrum of the unbleached solution, curve $B$ is the spectrum of the same solution after bleaching, and curve $C$ is the differential spectrum.

Therefore, in the following experiment the differential spectrum was measured at $500 \mathrm{~m} \mu$ only.

Homogenate of 40 whole bleached retinas was divided into four portions and DPN, homogenate of 10 pigment layers, homogenate of 10 pigment layers 
supplemented with DPN, and homogenate of 20 pigment layers supplemented with DPN were added to each portion. The differential spectra at $500 \mathrm{~m} \mu$ of the digitonin extract of each preparation after overnight incubation were 0.024 , $0.034,0.057$, and 0.0705 respectively.

In this preliminary experiment the result of Wald and Hubbard (10) was confirmed.

Action of acetylcholine, arecoline, and pilocarpine on the visual purple regeneration in vitro. The mixture of homogenate of 20 whole bleached retinas, homogenate of 20 pigment layers, and DPN supplemented with niacin amide was divided into two portions and buffer was added to one portion (served as control) and acetylcholine supplemented with eserine $1 /(100 \mathrm{r} / \mathrm{ml}$. $)$, arecoline or pilocarpine to another portion. There was almost no difference in the amounts of regenerated visual purple between control and the samples added with arecoline or pilocarpine and only a slight augmented regeneration was observed in the sample added with $0.1 \%$ acetylcholine (table 1 ).

TABLE 1. Action of Acetylcholine, Arecolin, and Pilocarpine on the Regeneration of Visual Purple in vitro

\begin{tabular}{|c|c|c|c|c|c|c|c|c|c|c|c|c|}
\hline \multirow{2}{*}{ 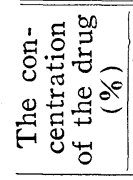 } & \multicolumn{4}{|c|}{ Acetylcholine } & \multicolumn{4}{|c|}{ Arecoline } & \multicolumn{4}{|c|}{ Pilocarpine } \\
\hline & 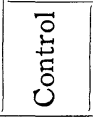 & 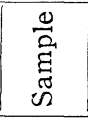 & 离导 & 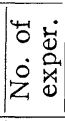 & 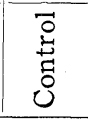 & $\begin{array}{l}\frac{0}{a} \\
\text { 范 } \\
\text { 品 }\end{array}$ & 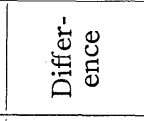 & 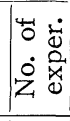 & 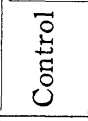 & $\begin{array}{l}\stackrel{0}{\Xi} \\
\stackrel{\Xi}{\pi} \\
\tilde{\Omega}\end{array}$ & 岂 岕 & 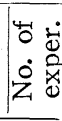 \\
\hline 0.0025 & 0.0735 & 0.0715 & $\begin{array}{l}-0.002 \\
(-2.7 \%)\end{array}$ & 3 & & & & & & & & \\
\hline 0.005 & 0.0561 & 0.0542 & $\begin{array}{c}-0.0019 \\
(-3.4 \%)\end{array}$ & 6 & & & & & & & & \\
\hline 0.01 & 0.0525 & 0.0518 & $\begin{array}{c}-0.0007 \\
(-1.3 \%)\end{array}$ & 3 & 0.0567 & 0.0574 & $\begin{array}{c}+0.0007 \\
(+1.2 \%)\end{array}$ & 3 & 0.0488 & 0.0445 & $\begin{array}{c}-0.0043 \\
(-8.8 \%)\end{array}$ & 3 \\
\hline 0.05 & 0.0500 & 0.0535 & $\begin{array}{c}+0.0035 \\
(+7.0 \%)\end{array}$ & 3 & & & & & & & & \\
\hline 0.1 & 0.0574 & 0.0645 & $\begin{array}{l}+0.0071 \\
(+12.4 \%)\end{array}$ & 5 & 0.0665 & 0.0594 & $\begin{array}{l}-0.0071 \\
(-10.7 \%)\end{array}$ & 3 & 0.0488 & 0.0500 & $\begin{array}{l}+0.0012 \\
(+2.5 \%)\end{array}$ & 3 \\
\hline 1.0 & & & & & & & & & 0.0481 & 0.0434 & $=\begin{array}{r}-0.0047 \\
(-9.8 \%)\end{array}$ & 3 \\
\hline
\end{tabular}

* indicates the density of regenerated visual purple.

Care was taken to adjust $p H$ to be equal in the control and in the sample.

Action of the pigment layer of toad injected with acetylcholine, arecoline, or pilocarpine on the visual purple regeneration. Homogenate of 20 whole bleached retinas of normal toads supplemented with DPN and niacin amide was divided into two portions and homogenate of 20 pigment layers of dark adapted normal toads was added to one portion (served as control) and homogenate of 20 pigment layers of dark adapted toads to which acetylcholine $(0.2 \%, 1.0 \mathrm{ml}$.), arecoline $(0.2 \%, 0.5 \mathrm{ml}$.$) or pilocarpine (2 \%, 0.5 \mathrm{ml}$.) was injected 60 to 90 minutes previously, to another portion. The amount of regenerated visual purple was higher in the sample added the pigment layer of toad injected with acetylcholine, arecoline, or pilocarpine than in the control (table 2, A).

If the pigment layer homogenate was boiled at $100^{\circ} \mathrm{C}$. for 3 minutes, the 
augmentative action of the pigment layer of toad injected with acetylcholine on the visual purple regeneration disappeared (table $2, \mathrm{~B}$ ).

TABLE 2. The Potency of the Pigment Layers after the Injection of Acetylcholine, Arecoline, or Pilocarpine for the Regeneration of Visual Purple

\begin{tabular}{|c|c|c|c|c|c|c|c|c|c|c|c|c|}
\hline & \multicolumn{4}{|c|}{ Acetylcholine } & \multicolumn{4}{|c|}{ Arecoline } & \multicolumn{4}{|c|}{ Pilocarpine } \\
\hline & \begin{tabular}{l}
$\overrightarrow{0}$ \\
\multirow{H}{0}{} \\
0 \\
0
\end{tabular} & 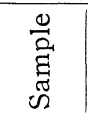 & 离㤩 & $\left|\begin{array}{ll}4 & \dot{0} \\
0 & \dot{0} \\
0 & 0 \\
\dot{x} & 0\end{array}\right|$ & \begin{tabular}{l}
$\overrightarrow{0}$ \\
\multirow{2}{*}{} \\
0 \\
0 \\
0
\end{tabular} & 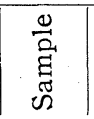 & 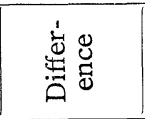 & 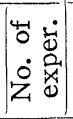 & $\begin{array}{l}\overrightarrow{0} \\
0 \\
0 \\
0 \\
0\end{array}$ & 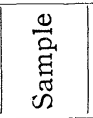 & 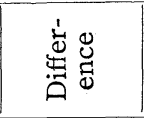 & $\begin{array}{ll}\text { 䓃 } & \dot{\vec{d}} \\
\dot{0} & \stackrel{0}{x} \\
z & 0\end{array}$ \\
\hline A & $0.0560^{*}$ & 0.0687 & $\begin{array}{c}+0.0127 \\
(+22.7 \%)\end{array}$ & 3 & 0.0437 & 0.0538 & $\left|\begin{array}{c}+0.0101 \\
(+23.1 \%)\end{array}\right|$ & 4 & 0.0521 & 0.0611 & $\left(\begin{array}{c}+0.009 \\
(+17.3 \%)\end{array}\right.$ & 3 \\
\hline B & 0.0280 & 0.0275 & $\begin{array}{l}-0.0005 \\
(-2.8 \%)\end{array}$ & 3 & & & & & & & & \\
\hline
\end{tabular}

* indicates the density of regenerated visual purple.

The homogenate of pigment layers was not heated and heated $\left(100^{\circ} \mathrm{C} ., 3\right.$ minutes) before adding to the reaction mixture in $\mathrm{A}$ and $\mathrm{B}$ respectively. The wet weight of the pigment layers of control and sample was adjusted to be the same.

\section{DISCUSSION}

Arecoline and pilocarpine have no augmentative action on the regeneration of visual purple in vitro, so it may be said that the augmentative action of these drugs on the visual purple regeneration is dependent on living process. Acetylcholine in very high concentration $(0.1 \%)$ has only a slight augmentative action on the visual purple regeneration in vitro, but such direct action does not seem to play any important part in vivo, because such high concentration of acetylcholine could not be induced in the retina after the injection of 0.3-1.0 $\mathrm{ml}$. of $0.1 \%$ or $1.0 \mathrm{ml}$. of $0.2 \%$ acetylcholine. Therefore the acceleratory action of acetylcholine on the visual purple regeneration in vivo seems to be also dependent on living process.

The potency of pigment layer for visual purple regeneration increased after the injection of acetylcholine, arecoline or pilocarpine, Therefore, the mode of action of these drugs on the visual purple regeneration seems not to be due to physical changes in the pigment layer but to chemical changes. The increased potency of the pigment layer seems to be due to increased potency of heat labile factor in the pigment layer, because it was not observed after boiling at $100^{\circ} \mathrm{C}$. for 3 minutes.

Kühne (1) postulated secretory theory and said a specific substance "Rhodophyllin" to be secreted from the pigment epithelium during visual purple regeneration, and the augmentative action of pilocarpine on the regeneration of visual purple was considered to be a powerful support for his theory. But there are many drug actions which cannot be explained by secretory theory. For example atropine has no inhibitory effect $(4,5)$, eserine has no acceleratory action $(4,5)$, and scopolamine (5) and methionine (13) have augmentative action on the regeneration of visual purple.

According to recent investigations the process of visual purple regeneration is not a simple reaction but complicated phenomena which involve many reac- 
tions (retinene reductase and heat labile factor reported by Wald and Hubbard (10), phosphorylation mentioned by Morton et al. (11), and pigment layer factor reported by Bliss (12)). Thus the mode of action of all augmentative drugs seems not to be the same and they would be classified into many groups according to their mode of action. The mode of action of acetylcholine, arecoline, and pilocarpine on the visual purple regeneration seems to belong to the same category, but it cannot be decided by present data whether the increased potency of the pigment layer after the injection of acetylcholine, arecoline, or pilocarpine is due to increased secretion or not. Such problem awaits further experimentation.

\section{CONCLUSION}

1) Arecoline and pilocarpine have no augmentative action on the regeneration of visual purple in vitro.

2) Acetylcholine in very high concentration $(0.1 \%)$ has only a slight augmentative effect on the visual purple regeneration in vitro.

3) The potency of pigment layer for visual purple regeneration increases after the injection of acetylcholine, arecoline, or pilocarpine.

4) The increased potency of pigment layer after the injection of acetylcholine was not observed, if the pigment layer is boiled at $100^{\circ} \mathrm{C}$. for 3 minutes.

5) The mode of action of acetylcholine, arecoline, and pilocarpine on the visual purple regeneration in vivo was discussed.

\section{REFERENCES}

1. Ayres, W. C. AND W. KüHne. Unters. Physiol. Inst. Heidelberg 2: 215, 1878.

2. DRESSER, H. Z. Biol. 22 : 23. 1886.

3. InAtomi, M. Chuo Ganka Tho (Japanese) $20: 283,1928$.

4. Amenomiya, J. Acta Ophthal. Jap. $34: 1459.1930$.

5. Hosoy A, Y. Jap. J. Physiol. (Japanese.) 8: 499, 1942.

6. BAll, S., GoOdWIN, T. W. AND R. A. MORTON. Biochem. J. 40 : lix, 1946.

7. Ball, S., Glover, J., Goodwin, T. W. AND R. A. Morton. ibid. 41: 29, 1947.

8. WALD, G. AND R. HubBard. J. Gen. Physiol. $32: 367,1948-49$.

9. Bliss, A. J. Biol. Chem. 172: 165, 1948; Biol. Bull. $97: 221,1949$; 99 : 330, 1950.

10. Hubbard, R. AND G. WAld. Proc. Natl. Acad. Sci. $37: 69,1950$.

11. Collins, F. D. AND R. A. MORTON. Biochem. J. 53: 152, 1953.

12. Bliss, A. J. Biol. Chem. $193: 525,1951$.

13. Huang, T. F. Jap. J. Physiol. $1: 165,1950$. 